\title{
Bronchopleural fistula following a video-assisted thoracoscopic surgery lobectomy after neoadjuvant therapy of pembrolizumab: a case report and literature review
}

\author{
Min $\mathrm{Cao}^{1}$, Yujie Fu ${ }^{1}$, Xiuying Xiao ${ }^{2}$, Jian Tang ${ }^{1}$, Daoqiang Tang ${ }^{3}$, Xiaojing Zhao ${ }^{1}$ \\ ${ }^{1}$ Department of Thoracic Surgery, Renji Hospital, Shanghai Jiao Tong University School of Medicine, Shanghai, China; ${ }^{2}$ Department of Oncology, \\ Renji Hospital, Shanghai Jiao Tong University School of Medicine, Shanghai, China; ${ }^{3}$ Department of Pathology, Renji Hospital, Shanghai Jiao Tong \\ University School of Medicine, Shanghai, China \\ Correspondence to: Xiaojing Zhao. Department of Thoracic Surgery, Renji Hospital, Shanghai Jiao Tong University School of Medicine, 10th Floor, \\ Building 7, 160 Pujian Road, Pudong New Area, Shanghai 200127, China. Email: zhaoxiaojing@renji.com.
}

\begin{abstract}
Lung cancer has the highest morbidity and mortality worldwide among the malignant tumors. The treatment of lung cancer was difficult in the decade years. Currently, the advent of immune checkpoint inhibitors (ICIs) has changed the pattern of lung cancer treatment. Programmed death-ligand 1 (PD-L1) inhibitors, such as pembrolizumab, plus platinum-based chemotherapy is the preferred treatment modality for advanced or metastatic non-small cell lung cancer (NSCLC) patients. The success of pembrolizumab in lung cancer has increased interest in expanding the use of neoadjuvant treatment. Immune-related adverse events (irAEs) are the most common adverse reactions in immunotherapy. The balance between the benefits and risks is crucial in this setting. Video-assisted thoracoscopic surgery (VATS) is a routine procedure of resectable NSCLC treatment. It appears to favor low postoperative complication rate. The prolonged air leakage (PAL) is the most common complication after VATS, and the bronchopleural fistula (BPF) is the most serious complication. The management of adverse events after VATS, especially in the setting of immune therapy, need to be raised. Here, we report a case of BPF following VATS lobectomy, which firstly presented the treatment-related adverse event with neoadjuvant combination therapy of pembrolizumab plus cisplatin/pemetrexed. Literature on novel neoadjuvant immunotherapy was also reviewed in the present study.
\end{abstract}

Keywords: Neoadjuvant immunotherapy; pembrolizumab; bronchopleural fistula (BPF); video-assisted thoracoscopic surgery (VATS); lobectomy; lung cancer

Submitted Oct 23, 2020. Accepted for publication Dec 16, 2020.

doi: 10.21037/atm-20-7582

View this article at: http://dx.doi.org/10.21037/atm-20-7582

\section{Introduction}

Lung cancer is the leading cause of cancer mortality, and the 5 -year survival rate is close to $15 \%$ worldwide (1). The use of immunotherapy has improved the treatment for NSCLC. Pembrolizumab is an immune inhibitor, and has been the preferred protocol for advanced NSCLC patients with positive programmed death-ligand 1 (PD-L1) expression. Pembrolizumab monotherapy or combination pembrolizumab therapy has been found to significantly prolong progression free survival (PFS) and/or overall survival (OS) compared with chemotherapy alone in lung cancer (KEYNOTE189/KEYNOTE407). Therefore, it is important to understand the role of pembrolizumab in the neoadjuvant setting. In the present study, we report a case of an immune-related adverse event following 3 cycles of neoadjuvant treatment with pembrolizumab plus cisplatin/ pemetrexed. It is the first reported case of BPF following VATS lobectomy after neoadjuvant immunotherapy, and a novel anastomotic model that may decrease the occurrence rate of BPF is proposed. 

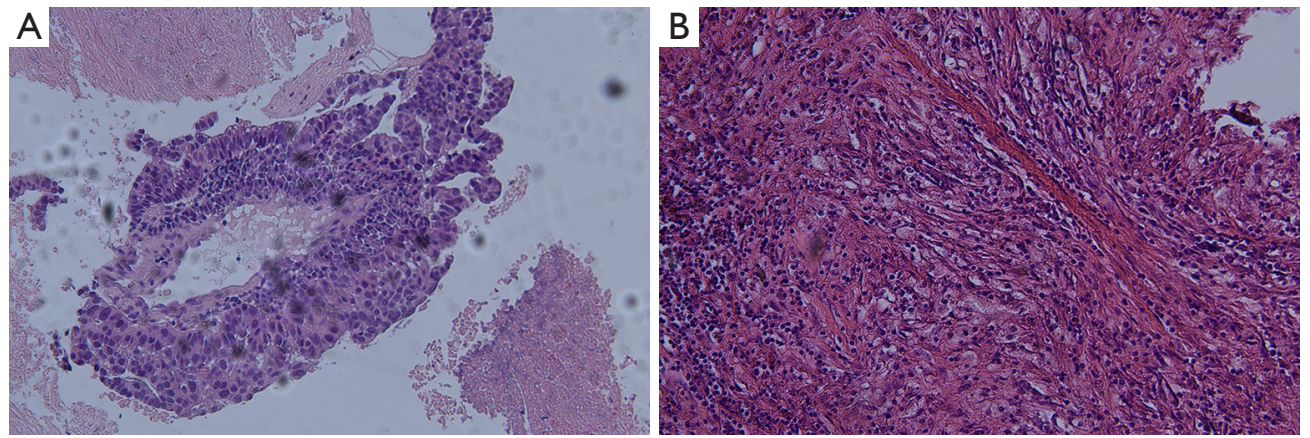

Figure 1 Tumor cells' morphology is showed by hematoxylin-eosin staining (A) Hematoxylin-eosin staining showing poorly differentiated adenocarcinoma and (B) numerous necrotic tumors after neoadjuvant immunotherapy. Original magnification $\times 100$.

We present the following article in accordance with the CARE reporting checklist (available at http://dx.doi. org/10.21037/atm-20-7582).

\section{Case presentation}

A 65-year old male patient presented to our institution (Department of Thoracic Surgery, Renji Hospital, Shanghai Jiao Tong University School of Medicine, Shanghai, China) complaining of cough for 2 months and symptom aggravation for 2 weeks without obvious inducement. Contrast-enhanced computed tomography (CT) scan showed a $48 \times 42 \mathrm{~mm}$ mass in the right upper lobe. Positron emission tomography-CT scan indicated mediastinal lymphatic metastasis in groups 2 and 4 . The low-differentiated adenocarcinoma was identified by CTguided puncture biopsy (Figure 1A). Immunohistochemistry included CK7(+), TFF-1(+), NaspinA(-), P40(-), P63(-), and $\mathrm{Ki}-67$ (30\%). PD-L1 tumor proportion score was $\geq 50 \%$; no epidermal growth factor receptor (EGFR) mutation and anaplastic lymphoma kinase (ALK) translocation were detected. The patient was diagnosed with lung adenocarcinoma (cT2bN2M0, stage IIIA).

The patient underwent 3 cycles of combination therapy of intravenous pembrolizumab $(200 \mathrm{mg})$ plus cisplatin $(130 \mathrm{mg}) /$ pemetrexed $(900 \mathrm{mg})$, and stopped medication 2 weeks prior to radical surgery. The therapeutic effect was evaluated as stable disease by Immune Response Evaluation Criteria in Solid Tumor. A radical right upper lobectomy was performed by video-assisted thoracoscopy ( 3 ports), and the patient had an uneventful recovery. A large number of necrotic tumor cells was found during the pathological examination (Figure 1B). Three weeks after the operation, the patient developed symptoms, including cough and fever (highest temperature was $38.8^{\circ} \mathrm{C}$ ). $\mathrm{CT}$ scan showed right hydro pneumothorax, and empyema was suspected. BPF was diagnosed by bronchoscopy. The patient was treated with conservative therapy. A drainage chest tube was immediately placed in the right chest cavity. All symptoms disappeared 1 day after drainage. Normal saline irrigation and antibiotic therapy were applied twice a day, and the fistula gradually closed 2 months later (Figure 2). At present, the patient has been followed up for 2 years without maintenance therapy. CT scan and blood tumor indicators were reviewed every 3 months. There was no recurrence of lung cancer.

Written informed consent was obtained from the patient for publication of this study and any accompanying images. All procedures performed in studies involving human participants were in accordance with the ethical standards of the institutional and/or national research committee(s) and with the Helsinki Declaration (as revised in 2013).

\section{Discussion}

The role of surgery in patients with stage IIIa-N2 NSCLC remains controversial. Pulmonary resection in patients with N2-positive lymph nodes is often performed by a multidisciplinary team. Previously published studies have reported the survival benefit of surgery after neoadjuvant treatment in resectable NSCLC patients. Patients with N2-negative lymph nodes after neoadjuvant therapy, in particular, have a favorable prognosis. Neoadjuvant therapy (including neoadjuvant chemoradiotherapy and neoadjuvant chemotherapy) is used in most institutions for patients who are candidates for surgery. However, the treatmentrelated toxicities of conventional neoadjuvant therapy can delay surgery and/or increase postoperative complications. The benefits of targeted therapy and immunotherapy in 

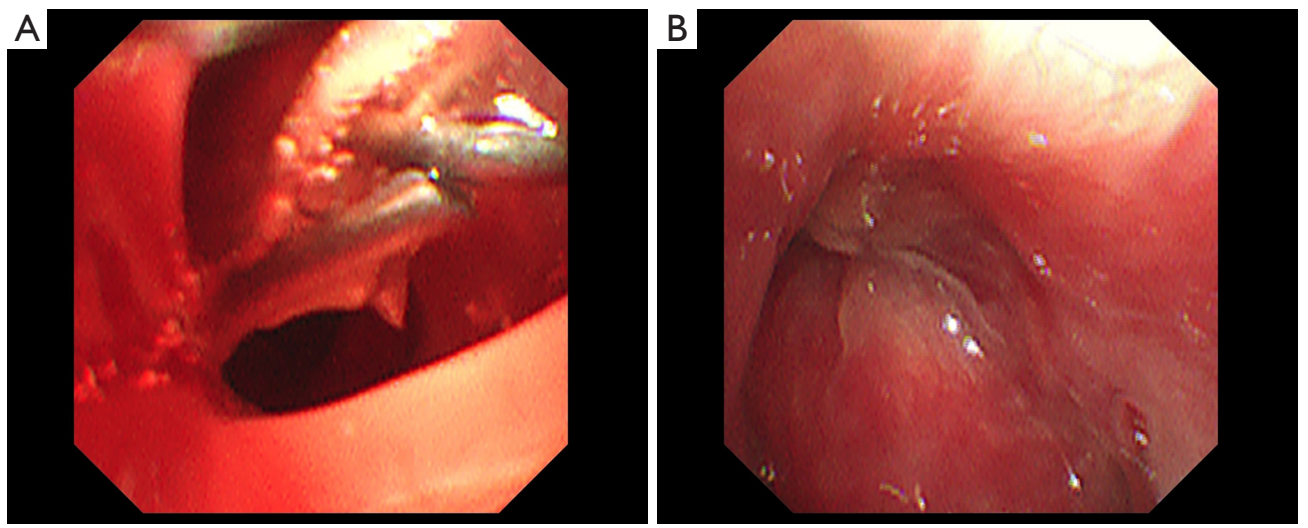

Figure 2 The bronchial stump is showed by bronchoscopy (A) The bronchial fistula of the right upper lobe and (B) bronchial fistula healing in the right upper lobe.

the neoadjuvant setting has been reported in case studies, including low toxicity and tolerance, and there is increasing interest in expanding their use (2-4).

Immunotherapy is a novel treatment option for locally advanced or metastatic NSCLC, and has been recommended by several previously published studies $(4,5)$. Pembrolizumab is an immune inhibitor, and is a humanized immunoglobulin G4 monoclonal antibody against PD-1. The KEYNOTE001 clinical trial demonstrated the safety, side-effects, and anti-tumor activity of pembrolizumab in patients with advanced NSCLC. The KEYNOTE010 trial reported that pembrolizumab significantly prolonged OS and major pathological response (MPR) in advanced or metastatic patients with positive PD-L1 expression, whose disease progressed after platinum-based chemotherapy. Remarkably, $>50 \%$ of the tumor proportion score (TPS) of PD-L1 expression is correlated with the improved efficacy of pembrolizumab (6). The KEYNOTE024 trial was designed to investigate the efficacy of pembrolizumab monotherapy versus platinum-based chemotherapy in patients with $>50 \%$ PD-L1 TPS expression. The significantly longer progression-free survival (10.3 vs. 6 months, $\mathrm{P}<0.001)$ and OS (30 vs. 14.2 months) confirmed the role of pembrolizumab as first-line therapy for patients with advanced or metastatic NSCLC. A randomized, open-label, phase 3 trail (KEYNOTE042) investigated the survival benefit of pembrolizumab monotherapy versus chemotherapy for patients with a PD-L1 TPS of $>1 \%$. The OS analysis suggested that patients without EGFR mutation or ALK translocation could benefit from pembrolizumab treatment for locally advanced or metastatic NSCLC (6). Pembrolizumab has been recommended by the US Food and Drug Administration as the first-line treatment for patient with PD-L1 expression.

The success of neoadjuvant immunotherapy in the treatment of various cancers suggests its potential benefit in the treatment of NSCLC. PD-1 inhibitor nivolumab has been administered in patients with untreated, surgically resectable early NSCLC, and was found to be safe and feasible, without any unreported treatment-related adverse events (7). Therefore, the use of PD-1 antibody pembrolizumab in the neoadjuvant setting is desired. A recently published study demonstrated that platinum-based chemotherapy enhanced T-cell cytotoxicity (8), which has the potential to activate anti-tumor activity. Combination therapy has been shown to make tumors highly responsive to immunotherapy via different pathways of tumor growth. We reported that neoadjuvant pembrolizumab plus cisplatin/pemetrexed would lead to MPR in tumors. Previously published studies have reported the high rate of MPR among patients undergoing neoadjuvant immunotherapy, which is consistent with our results (9); a high MPR has also been associated with increased tumor mutational burden (9). Although there is limited evidence, immunotherapy in the neoadjuvant setting is promising. Further studies are needed to verify that a high MPR is associated with survival benefits in NSCLC patients.

In this study, the patient has been followed up for 2 years without maintenance therapy, and there was no recurrence of disease. Immune maintenance therapy refers to use of ICIs after initial treatment, which has been proposed as either continuation maintenance or switch maintenance. According to NCCN Guidelines 2020, immune maintenance therapy plays an important role in 
A

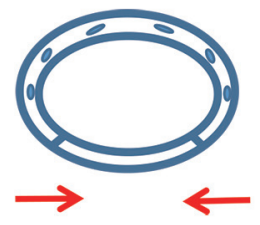

B

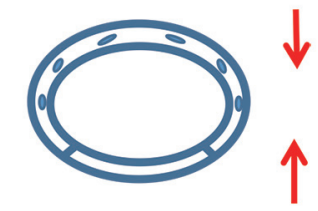

Figure 3 The diagram of anastomose. (A) Anastomose the right upper lobe bronchus via the fourth intercostal at the anterior axillary line and (B) anastomose via the seventh intercostal at the posterior axillary line.

advanced or metastatic NSCLC patients, which could prolong the PFS and OS in this NSCLC population (10). The best time for immune maintenance therapy is after 4-6 cycles of initial therapy. The patients should receive maintenance therapy for 2 years if they received front-line immunotherapy, or maintenance therapy until progression if they received second-line immunotherapy. During the period of maintenance therapy, different ICIs have different efficacy. However, a common point is the immune-related adverse events (irAEs), which are the most common adverse reactions. There are many guidelines on the management of irAEs have been published $(11,12)$. Different irAEs need different treatments according to the guidelines. In this condition, the most important thing is to balance the benefits with the potential risks. Another point is the expression levels of PD-L1 in maintenance therapy. Though the expression level of PD-L1 is positively correlated with the efficacy of Pembrolizumab in previously untreated NSCLC patients (6), the correlation in maintenance therapy is still unclear.

VATS is a standard procedure for early-stage NSCLC. It appears to favor lower short-term morbidity and improved long-term survival rates. Compared with thoracotomy, the application value of VATS is associated with shorter chest tube duration, shorter length hospital stay and lower cost of hospitalization (13).

The prolonged air leakage (PAL) is the most common complication after VATS $(5-15 \%)(14,15)$. The low FEV1\% of predicted, previous smoking history, pleural adhesions, and major lung resection are convincing risk factors for PAL after lung operation. The PAL may prolong the hospital stay and increase the hospitalization cost, the risk of empyema and some other possible cardiopulmonary complications.
$\mathrm{BPF}$ is one of the most serious postoperative adverse events, and is a potentially fatal complication of pulmonary resection. The overall $\mathrm{BPF}$ incidence is $4.4 \%$ and the mortality rate is $27.2 \%$ (16). Neoadjuvant therapy is considered a risk factor of BPF, and surgical procedure, right side, and age are mentioned as risk factors (17). Prompt management is necessary to treat BPF. In the present case, BPF was suspected by clinical symptoms, such as cough and fever, and was confirmed by bronchoscope. A drainage chest tube was immediately placed in the right chest cavity after the diagnosis of BPF. The management of this complication usually includes conservative, endoscopic, and surgical treatments. Fuso et al. reported that endoscopic therapy is a useful option in the management of BPF (18). Biologic or synthetic glues were used to seal the fistula. Sfyridis $e t$ al. reported that intercostal muscle flap coverage was significantly effective for the prevention of BPF (19). However, conservative therapy, including adequate drainage and appropriate antibiotic use, is considered safe and useful for post-lobectomy fistulas (20), which is consistent with our study. Combination therapy is more effective than monotherapy alone, and combination therapy has been shown to reduce the mean resolution time compared with monotherapy alone. More high-quality studies are needed to confirm the reliability of the novel management of $\mathrm{BPF}$. Conventional neoadjuvant therapy is a risk factors of $\mathrm{BPF}$, although there is no evidence suggesting that neoadjuvant immunotherapy might lead to BPF after pulmonary surgery. Careful preoperative assessment is necessary, as is postoperative systemic nutritional support. In our case, the right upper lobe bronchus was cut by a endoscopic linear cutter via the fourth intercostal at the anterior axillary line in the 3 -port procedure (Figure $3 A$ ). The bronchus membrane and cartilage are stapled together, which may increase the rate of BPF postoperatively. An alternative method of dealing with the right upper lobe bronchus is to locate the endoscopic linear cutter via the seventh intercostal at the posterior axillary line, which may anastomose the membrane and cartilage (Figure 3B). This may improve the healing of the bronchus stump and decrease the rate of postoperative BPF.

\section{Conclusions}

Pembrolizumab is the preferred treatment modality for advanced NSCLC patients whose PD-L1 TPS $\geq 50 \%$, and is safe and well tolerated. The use of pembrolizumab in the neoadjuvant setting is expected to increase in the 
future. Treatment-related adverse events, such as BPF, may hinder the use of pembrolizumab combination therapy in neoadjuvant immunotherapy. Correct anastomose may decrease the rate of BPF after pulmonary surgery. More high-level evidence is needed to confirm the safety and feasibility of pembrolizumab in the neoadjuvant setting.

\section{Acknowledgments}

Funding: None.

\section{Footnote}

Reporting Checklist: The authors have completed the CARE reporting checklist. Available at http://dx.doi.org/10.21037/ atm-20-7582

Conflicts of Interest: All authors have completed the ICMJE uniform disclosure form (available at http://dx.doi. org/10.21037/atm-20-7582). The authors have no conflicts of interest to declare.

Ethical Statement: The authors are accountable for all aspects of the work in ensuring that questions related to the accuracy or integrity of any part of the work are appropriately investigated and resolved. Written informed consent was obtained from the patient for publication of this study and any accompanying images. All procedures performed in studies involving human participants were in accordance with the ethical standards of the institutional and/or national research committee(s) and with the Helsinki Declaration (as revised in 2013).

Open Access Statement: This is an Open Access article distributed in accordance with the Creative Commons Attribution-NonCommercial-NoDerivs 4.0 International License (CC BY-NC-ND 4.0), which permits the noncommercial replication and distribution of the article with the strict proviso that no changes or edits are made and the original work is properly cited (including links to both the formal publication through the relevant DOI and the license). See: https://creativecommons.org/licenses/by-nc-nd/4.0/.

\section{References}

1. Herbst RS, Heymach JV, Lippman SM. Lung cancer. N Engl J Med 2008;359:1367-80.

2. Cao M, Zhao X, Yin H, et al. Video-assisted thoracoscopic surgery right upper lobectomy after neoadjuvant targeted therapy. J Thorac Dis 2019;11:252-4.

3. Yeh J, Marrone KA, Forde PM. Neoadjuvant and consolidation immuno-oncology therapy in stage III nonsmall cell lung cancer. J Thorac Dis 2018;10:S451-9.

4. Garon EB, Rizvi NA, Hui R, et al. Pembrolizumab for the treatment of non-small-cell lung cancer. N Engl J Med 2015;372:2018-28.

5. Rittmeyer A, Barlesi F, Waterkamp D, et al. Atezolizumab versus docetaxel in patients with previously treated non-small cell lung cancer(OAK): a phase 3, openlabel, multicentre randomised controlled trail. Lancet 2017;389:255-65.

6. Mok TSK, Wu YL, Kudaba I, et al. Pembrolizumab versus chemotherapy for previously untreated, PD-L1expressing, locally advanced or metastatic non-small-cell lung cancer (KEYNOTE-042): a randomised, open-label, controlled, phase 3 trial. Lancet 2019;393:1819-30.

7. Forde PM, Chaft JE, Smith KN, et al. Neoadjuvant PD-1 Blockade in Resectable Lung Cancer. N Engl J Med 2018;378:1976-86.

8. Apetoh L, Ladoire S, Coukos G, et al. Combining immunotherapy and anticancer agents: the right path to achieve cancer cure? Ann Oncol 2015;26:1813-23.

9. Lommatzsch M, Bratke K, Stoll P. Neoadjuvant PD-1 Blockade in Resectable Lung Cancer. N Engl J Med 2018;379:e14.

10. Murakami S. Durvalumab for the treatment of nonsmall cell lung cancer. Expert Rev Anticancer Ther 2019;19:1009-16.

11. Puzanov I, Diab A, Abdallah K, et al. Society for Immunotherapy of Cancer Toxicity Management Working Group. Managing toxicities associated with immune checkpoint inhibitors: consensus recommendations from the Society for Immunotherapy of Cancer (SITC) Toxicity Management Working Group. J Immunother Cancer 2017;5:95.

12. Thompson JA, Schneider BJ, Brahmer J, et al. NCCN Guidelines Insights: Management of ImmunotherapyRelated Toxicities, Version 1.2020. J Natl Compr Canc Netw 2020;18:230-41.

13. Whitson BA, Groth SS, Duval SJ, et al. Surgery for early-stage non-small cell lung cancer: a systematic review of the video-assisted thoracoscopic surgery versus thoracotomy approaches to lobectomy. Ann Thorac Surg 2008;86:2008-16.

14. Bendixen M, Jørgensen OD, Kronborg C, et al. Postoperative pain and quality of life after lobectomy 
via video-assisted thoracoscopic surgery or anterolateral thoracotomy for early stage lung cancer: a randomised controlled trial. Lancet Oncol 2016;17:836-44.

15. McKenna RJ Jr. New approaches to the minimally invasive treatment of lung cancer. Cancer J 2005;11:73-6.

16. Sirbu H, Busch T, Aleksic I, et al. Bronchopleural fistula in the surgery of non-small cell lung cancer: incidence, risk factors, and management. Ann Thorac Cardiovasc Surg 2001;7:330-6.

17. Hollaus PH, Lax F, El-Nashef BB, et al. Natural history of bronchopleural fistula after pneumonectomy: a review of 96 cases. Ann Thorac Surg 1997;63:1391-6; discussion

Cite this article as: Cao M, Fu Y, Xiao X, Tang J, Tang D, Zhao X. Bronchopleural fistula following a video-assisted thoracoscopic surgery lobectomy after neoadjuvant therapy of pembrolizumab: a case report and literature review. Ann Transl Med 2020;8(24):1691. doi: 10.21037/atm-20-7582
1396-7.

18. Fuso L, Varone F, Nachira D, et al. Incidence and Management of Post-Lobectomy and Pneumonectomy Bronchopleural Fistula. Lung 2016;194:299-305.

19. Sfyridis PG, Kapetanakis EI, Baltayiannis NE, et al. Bronchial stump buttressing with an intercostal muscle flap in diabetic patients. Ann Thorac Surg 2007;84:967-71.

20. Cooper WA, Miller JI Jr. Management of bronchopleural fistula after lobectomy. Semin Thorac Cardiovasc Surg 2001;13:8-12.

(English Language Editor: R. Scott) 